\title{
A ratio-dependent food chain model and its applications to biological control
}

\author{
Sze-Bi Hsu ${ }^{\text {a,1 }}$, Tzy-Wei Hwang b,1, Yang Kuang c,* \\ a Department of Mathematics, National Tsing Hua University, Hsinchu, Taiwan, ROC \\ b Department of Mathematics, Kaohsiung Normal University, 802, Kaohsiung, Taiwan, ROC \\ c Department of Mathematics, Arizona State University, Tempe, AZ 85287-1804, USA
}

Received 8 January 2002; received in revised form 11 June 2002; accepted 27 June 2002

\begin{abstract}
While biological controls have been successfully and frequently implemented by nature and human, plausible mathematical models are yet to be found to explain the often observed deterministic extinctions of both pest and control agent in such processes. In this paper we study a three trophic level food chain model with ratio-dependent Michaelis-Menten type functional responses. We shall show that this model is rich in boundary dynamics and is capable of generating such extinction dynamics. Two trophic level MichaelisMenten type ratio-dependent predator-prey system was globally and systematically analyzed in details recently. A distinct and realistic feature of ratio-dependence is its capability of producing the extinction of prey species, and hence the collapse of the system. Another distinctive feature of this model is that its dynamical outcomes may depend on initial populations levels. Theses features, if preserved in a three trophic food chain model, make it appealing for modelling certain biological control processes (where prey is a plant species, middle predator as a pest, and top predator as a biological control agent) where the simultaneous extinctions of pest and control agent is the hallmark of their successes and are usually dependent on the amount of control agent. Our results indicate that this extinction dynamics and sensitivity to initial population levels are not only preserved, but also enriched in the three trophic level food chain model. Specifically, we provide partial answers to questions such as: under what scenarios a potential biological control may be successful, and when it may fail. We also study the questions such as what conditions ensure the coexistence of all the three species in the forms of a stable steady state and limit cycle, respectively. A multiple attractor scenario is found.
\end{abstract}

(C) 2003 Elsevier Science Inc. All rights reserved.

AMS: 34D23; 34D45; 92D25

${ }^{*}$ Corresponding author. Tel.: +1-480 965 6915; fax: +1-480 9658119.

E-mail address: kuang@asu.edu (Y. Kuang).

${ }^{1}$ Research supported by National Council of Science, Republic of China. 
Keywords: Biological control; Predator-prey model; Limit cycle; Chaos; Extinction; Food chain model; Ratiodependence; Simple food chain

\section{Introduction}

Biological control is, generally, man's use of a specially chosen living organism to control a particular pest. This chosen organism might be a predator, parasite, or disease which will attack the harmful insect. It is a form of manipulating nature to increase a desired effect. A complete biological control program may range from choosing a pesticide which will be least harmful to beneficial insects, to raising and releasing one insect to have it attack another, almost like a 'living insecticide'. In short, biological control is a tool to be considered in constructing an integrated pest management scheme for protected plant production. It may also be a more economical alternative to some insecticides. Some biological control measures can actually prevent economic damage to agricultural crops. Unlike most insecticides, biological controls are often very specific for a particular pest. Other helpful insects, animals, or people can go completely unaffected or disturbed by their use.

In this paper we shall study a three trophic level simple food chain model with ratio-dependence and Michaelis-Menten (or Holling type II) functional response and its applications to biological control. Before we introduce the model, we would like to present a brief historical account of the biological relevance of two different types of predator-prey models: the classical prey-dependent ones and the controversial [1,2] ratio-dependent ones.

The classical prey-dependent predator-prey system often takes the general form of

$$
\begin{aligned}
& x^{\prime}(t)=x g(x)-c y p(x), \\
& y^{\prime}(t)=(p(x)-d) y,
\end{aligned}
$$

where $x, y$ stand for prey and predator density, respectively. $p(x)$ is the so-called predator functional response and $c, d>0$ are the conversion rate and predator's death rate respectively. If $p(x)=(m x / a+x), g(x)=r(1-(x / K))$, then (1.1) becomes the following well-known predatorprey model with Michaelis-Menten functional response [3,4]:

$$
\begin{aligned}
& x^{\prime}(t)=r x\left(1-\frac{x}{K}\right)-c \frac{m x y}{a+x}, \quad x(0)>0, \\
& y^{\prime}(t)=\left(\frac{m x}{a+x}-d\right) y, \quad y(0)>0,
\end{aligned}
$$

where $r, K, a, m$ are positive constants that stand for prey intrinsic growth rate, carrying capacity, half saturation constant, maximal predator growth rate, respectively. This model exhibits the wellknown 'paradox of enrichment' formulated by Hairston et al. [5] and Rosenzweig [6] which states that according to model (1.2), enriching a predator-prey system (increasing the carrying capacity $K$ ) will cause an increase in the equilibrium density of the predator but not in that of the prey, and will destabilize the positive equilibrium (the positive steady state changes from stable to unstable as $K$ increases) and thus increases the possibility of stochastic extinction of predator. Unfortunately, numerous field observations provide contrary to this 'paradox of enrichment'. What often 
observed in nature is that fertilization does increases the prey density, does not destabilize a stable steady state and fails to increase the amplitude of oscillations in systems that already cycle [7].

A variation of the 'paradox of enrichment' is the so called 'biological control paradox', which was recently brought into discussion by Luck [8], stating that according to (1.2), we cannot have both a low and stable prey equilibrium density. However, in reality, there are numerous examples of successful biological control where the prey are maintained at densities less than $2 \%$ of their carrying capacities [9]. This clearly indicates that the paradox of biological control is not intrinsic to predator-prey interactions. Another noteworthy prediction from model (1.1) is that prey and predator species can not extinct simultaneously (mutual extinction). This, however, clearly contradicts Gause's classic observation of mutual extinction in the protozoans, Paramecium and its predator Didinium [2,10].

Recently there is a growing evidences [11-13] that in some situations, especially when predator have to search for food (and therefore have to share or compete for food), a more suitable general predator-prey theory should be based on the so called ratio-dependent theory, which can be roughly stated as that the per capita predator growth rate should be a function of the ratio of prey to predator abundance. This is supported by numerous field and laboratory experiments and observations [11,14]. Generally, a ratio-dependent predator-prey model takes the form

$$
\begin{aligned}
& x^{\prime}(t)=x f(x)-\operatorname{cyp}(x / y), \quad x(0)>0, \\
& y^{\prime}(t)=(p(x / y)-d) y, \quad y(0)>0 .
\end{aligned}
$$

If $p(x)=(m x / a+x), f(x)=r(1-(x / K))$ then (1.3) becomes a ratio-dependent predator-prey model with Michaelis-Menten functional response:

$$
\begin{aligned}
& x^{\prime}(t)=r x\left(1-\frac{x}{K}\right)-c \frac{m x y}{x+a y}, \quad x(0)>0, \\
& y^{\prime}(t)=\left(\frac{m x}{x+a y}-d\right) y, \quad y(0)>0 .
\end{aligned}
$$

System (1.4) was studied in details by Hsu, Hwang and Kuang [15], Kuang and Beretta [16], Jost et al. [17], Berezovskaya et al. [18], Xiao and Ruan [19], Freedman and Mathsen [20], and others. Geometrically, the differences of prey-dependent model (1.2) and ratio-dependent model (1.4) are obvious, the former has a vertical predator isocline, while the latter has a slanted one passing through the origin. There are more differences in their prey isoclines. The analysis of (1.4) by Hsu, Hwang and Kuang [15] shows that the ratio-dependent models are capable of producing far richer and biologically more realistic dynamics. Specifically, it will not produce the paradox of biological control and the paradox of enrichment. It also allows mutual extinction as a possible outcome of a given predator-prey interaction [16,17]. For some other models relevant to ratio dependence, the interested are referred to [21,22].

For the mathematical models of multiple species interaction, we studied a model of two predators competing for a single prey with ratio-dependence in [23]. Another important mathematical model of multiple species interaction is the so-called food chain model. In the paper of Freedman and Waltman [24], the authors studied the persistence of a classical (i.e. prey-dependent) three species food chain model. Chiu and Hsu [25] discussed the extinction of top predator in a classical three-level food chain model with Michaelis-Menten functional response. Hastings and Powell [26], Klebanoff and Hastings [27,28], McCann and Yodzis [29], Kuznetsov and Rinaldi 
[30], Muratori and Rinaldi [31] and others studied structures relevant to chaos in three species classical food chains. Freedman and So [32] studied the global stability and persistence of a simple but general food chain model. Kuang [33] studied similar questions for a diffusive version of that simple food chain model. All these prey-dependent models, while mathematically interesting, inherit the mechanism that generates the factitious paradox of enrichment and fail to produce realistic extinction dynamics such as the collapse of the system.

Mathematical models of many biological control processes naturally call for differential systems with three equations describing the growth of plant, pest and top predator (control agent), respectively. The interaction of these three species often forms a simple food chain. Clearly, the classical food chain model is ill suited for this task. Indeed, researchers that adopt the classical prey-dependent models are forced to resort to stochastic effects to explain the frequently observed deterministic mutual extinctions of host (pest) and control agent (predator or parasite of the host) [34]. This motivates us to consider the following three trophic level food chain model with ratiodependence:

$$
\begin{aligned}
& x^{\prime}(t)=r x\left(1-\frac{x}{K}\right)-\frac{1}{\eta_{1}} \frac{m_{1} x y}{a_{1} y+x}, \quad x(0)>0, \\
& y^{\prime}(t)=\frac{m_{1} x y}{a_{1} y+x}-d_{1} y-\frac{1}{\eta_{2}} \frac{m_{2} y z}{a_{2} z+y}, \quad y(0)>0, \\
& z^{\prime}(t)=\frac{m_{2} y z}{a_{2} z+y}-d_{2} z, \quad z(0)>0,
\end{aligned}
$$

where $x, y, z$ stand for the population density of prey, predator and top predator, respectively. For $i=1,2, \eta_{i}, m_{i}, a_{i}, d_{i}$ are the yield constants, maximal predator growth rates, half-saturation constants and predator's death rates respectively. $r$ and $K$, as before, are the prey intrinsic growth rate and carrying capacity respectively. Observe that the simple relation of these three species: $z$ prey on $y$ and only on $y$, and $y$ prey on $x$ and nutrient recycling is not accounted for. This simple relation produces the so-called simple food chain. A distinct feature of simple food chain is the socalled domino effect: if one species dies out, all the species at higher trophic level die out as well.

For simplicity, we non-dimensionalizes the system (1.5) with the following scaling:

$$
\begin{aligned}
& t \rightarrow r t, \quad x \rightarrow \frac{x}{K}, \quad y \rightarrow \frac{a_{1}}{K} y, \quad z \rightarrow \frac{a_{2} a_{1}}{K} z, \\
& m_{1} \rightarrow \frac{m_{1}}{r}, \quad d_{1} \rightarrow \frac{d_{1}}{r}, \quad m_{2} \rightarrow \frac{m_{2}}{r}, \quad d_{2} \rightarrow \frac{d_{2}}{r},
\end{aligned}
$$

then the system (1.5) takes the form

$$
\begin{aligned}
& x^{\prime}(t)=x(1-x)-\frac{c_{1} x y}{x+y}=F_{1}(x, y), \quad x(0)>0, \\
& y^{\prime}(t)=\frac{m_{1} x y}{x+y}-d_{1} y-\frac{c_{2} y z}{y+z}=F_{2}(x, y, z), \quad y(0)>0, \\
& z^{\prime}(t)=\frac{m_{2} y z}{y+z}-d_{2} z=F_{3}(y, z), \quad z(0)>0,
\end{aligned}
$$

where

$$
c_{1}=\frac{m_{1}}{\eta_{1} a_{1} r}, \quad c_{2}=\frac{m_{2}}{\eta_{2} a_{2} r} .
$$


We note that in (1.6), the functions $F_{i}(x, y, z), i=1,2,3$ are defined for $x>0, y>0, z>0$. Obviously

$$
\begin{aligned}
& \lim _{(x, y) \rightarrow(0,0)} F_{1}(x, y)=0, \\
& \lim _{(x, y, z) \rightarrow(0,0,0)} F_{2}(x, y, z)=0,
\end{aligned}
$$

and

$$
\lim _{(y, z) \rightarrow(0,0)} F_{3}(y, z)=0 .
$$

If we extend the domain of $F_{i}(x, y, z)$ to $\{(x, y, z): x \geqslant 0, y \geqslant 0, z \geqslant 0\}$ by $(1.8)$, then $(0,0,0)$ is an equilibrium of (1.6), and the stable manifold of $(0,0,0)$ contains the two-dimensional set $\{(x, y, z): x=0, y \geqslant 0, z \geqslant 0\}$.

In this paper we shall answer the following questions: What may cause the extinction of the top predator? What promotes the coexistence of all the three species? Do they coexist in the form of steady state or oscillation? How the outcomes depend on initial populations? When a biological control may succeed, and when it may fail?

The rest of this paper is organized as follows. In Section 2, we find the necessary and sufficient conditions for the existence of interior equilibrium $E_{c}=\left(x_{c}, y_{c}, z_{c}\right)$. We show that if $E_{c}$ does not exist then the top predator goes to extinction. We also provide scenarios when biological control is feasible and when it may fail. In Section 3, we study coexistence state and the stability of the interior equilibrium $E_{c}$. In Section 4, we discuss various scenarios where the outcomes depend on the initial populations. In particular, we found a tri-stability scenario that distinct solutions can be attracted to the origin, pest free steady state and positive steady state simultaneously for the same set of parameter. Section 5 presents additional biological implications of our mathematical findings and proposes some well motivated mathematical questions for future study. Throughout this manuscript, extensive graphical and computational works are presented to illustrate our mathematical observations and findings.

\section{Extinction scenarios}

In this section we study the asymptotic behavior of the solution of the following non-dimensional three species food chain model with ratio-dependence:

$$
\begin{aligned}
& x^{\prime}(t)=x(1-x)-\frac{c_{1} x y}{x+y}, \quad x(0)>0, \\
& y^{\prime}(t)=\frac{m_{1} x y}{x+y}-d_{1} y-\frac{c_{2} y z}{y+z}, \quad y(0)>0, \\
& z^{\prime}(t)=\frac{m_{2} y z}{y+z}-d_{2} z, \quad z(0)>0 .
\end{aligned}
$$

We shall examine conditions that render certain species extinct. Scenarios include the extinction of species $x$ (and hence $y$ and $z$ ), the extinction of $y$ (and hence $z$ ) but not $x$, the extinction of top predator $z$ (but not $x$ and $y$ ). 
First we consider the existence and uniqueness of the interior equilibrium $E_{c}=\left(x_{c}, y_{c}, z_{c}\right)$, $x_{c}, y_{c}, z_{c}>0$. From Eq. (2.3), $y_{c}, z_{c}$ satisfy

$$
\frac{m_{2} y_{c}}{y_{c}+z_{c}}-d_{2}=0
$$

or

$$
z_{c}=\frac{m_{2}-d_{2}}{d_{2}} y_{c}
$$

From Eq. (2.1), $x_{c}, y_{c}$ satisfy

$$
\left(1-x_{c}\right)-\frac{c_{1} y_{c}}{x_{c}+y_{c}}=0
$$

or

$$
y_{c}=\frac{x_{c}\left(1-x_{c}\right)}{c_{1}-\left(1-x_{c}\right)} .
$$

From Eq. (2.2), $x_{c}, y_{c}, z_{c}$ satisfy

$$
\frac{m_{1} x_{c}}{x_{c}+y_{c}}-d_{1}=\frac{c_{2} z_{c}}{z_{c}+y_{c}},
$$

substituting (2.4) into (2.6) yields

$$
\frac{m_{1} x_{c}}{x_{c}+y_{c}}-d_{1}=c_{2} \frac{m_{2}-d_{2}}{m_{2}},
$$

or

$$
y_{c}=(A-1) x_{c},
$$

where

$$
A=\frac{m_{1}}{c_{2}\left(\left(m_{2}-d_{2}\right) / m_{2}\right)+d_{1}} .
$$

Substituting (2.7) into (2.5) yields

$$
x_{c}=\frac{1}{A}\left(c_{1}+A\left(1-c_{1}\right)\right) .
$$

We have the following lemma.

Lemma 2.1. The interior equilibrium $E_{c}=\left(x_{c}, y_{c}, z_{c}\right)$ of the system (2.1)-(2.3) exists if and only if the following (i)-(iii) are satisfied:

(i) $m_{2}>d_{2}$,

(ii) $A>1$,

(iii) $0<c_{1}<A /(A-1)$.

Furthermore $x_{c}, y_{c}, z_{c}$ are given by (2.4), (2.7) and (2.9). 
Next we shall show that if the interior equilibrium $E_{c}$ does not exist then the top predator goes to extinction. Before we prove the extinction result, we need the following lemma.

Lemma 2.2. The solution $x(t), y(t), z(t)$ of (2.1)-(2.3) are positive and bounded for all $t \geqslant 0$.

Proof. Obviously the solutions $x(t), y(t), z(t)$ are positive for $t \geqslant 0$ and given any $\epsilon>0, x(t) \leqslant 1+\epsilon$ for $t$ sufficiently large. From (2.1), it follows that

$$
\begin{aligned}
\left(\frac{m_{1}}{c_{1}} x+y+\frac{m_{2}}{c_{2}} z\right)^{\prime} & =\frac{m_{1}}{c_{1}} x(1-x)-d_{1} y-d_{2} \frac{m_{2}}{c_{2}} z \\
& \leqslant \frac{m_{1}}{c_{1}} x-\min \left\{d_{1}, d_{2}\right\}\left(y+\frac{m_{2}}{c_{2}} z\right) \\
& \leqslant \xi-\min \left\{d_{1}, d_{2}\right\}\left(\frac{m_{1}}{c_{1}} x+y+\frac{m_{2}}{c_{2}} z\right),
\end{aligned}
$$

where $\xi=\left(m_{1} / c_{1}\right)\left(1+\min \left\{d_{1}, d_{2}\right\}\right)(1+\epsilon)$.

Then

$$
\frac{m_{1}}{c_{1}} x(t)+y(t)+\frac{m_{2}}{c_{2}} z(t) \leqslant \frac{\xi}{\min \left\{d_{1}, d_{2}\right\}}+\epsilon
$$

for $t$ sufficiently large. Hence we complete the proof of Lemma 2.2.

In the following lemma, we prove a straightforward result on the extinction of top predator. Namely, if the death rate of top predator is no less than its maximum birth rate, then it will face extinction.

Lemma 2.3. If $m_{2} \leqslant d_{2}$ then $\lim _{t \rightarrow \infty} z(t)=0$.

Proof. Observe that when $m_{2} \leqslant d_{2}$, we have $m_{2} y /(y+z)<m_{2}$, and hence $z^{\prime}(t)<0$. Therefore $\lim _{t \rightarrow \infty} z(t)$ exists and non-negative. We claim $\lim _{t \rightarrow \infty} z(t)=0$. Otherwise, there is a positive constant $\eta$, such that $\lim _{t \rightarrow \infty} z(t)=\eta$. Given $\eta>\epsilon>0$, there exists $t_{0}>0$, such that $\eta-\epsilon<$ $z(t)<\eta+\epsilon$ for $t \geqslant t_{0}$. In addition, there is a positive constant $y_{\max }$ such that $y(t)<y_{\max }$ for $t \geqslant t_{0}$. From Eq. (2.3), it follows that

$$
\begin{aligned}
z(t) & =z\left(t_{0}\right) \exp \left(\int_{t_{0}}^{t}\left(\frac{m_{2} y(s)}{y(s)+z(s)}-d_{2}\right) \mathrm{d} s\right) \\
& \leqslant z\left(t_{0}\right) \exp \left(\int_{t_{0}}^{t}\left(\frac{m_{2} y(s)}{y(s)+\eta-\epsilon}-d_{2}\right) \mathrm{d} s\right) \\
& \leqslant z\left(t_{0}\right) \exp \left(\frac{-(\eta-\epsilon) d_{2}}{y_{\max }+(\eta-\epsilon)}\left(t-t_{0}\right)\right) \rightarrow 0 \text { as } t \rightarrow \infty
\end{aligned}
$$

which is a contradiction under the assumption $m_{2} \leqslant d_{2}$.

Lemma 2.4. Let $m_{2}>d_{2}$ and $0<A \leqslant 1$ where $A$ is given by (2.8). Then $\lim _{t \rightarrow \infty} y(t)=0$ and $\lim _{t \rightarrow \infty} z(t)=0$. 
Proof. Since $0<A \leqslant 1$, from (2.8) it follows that $\left(c_{2} / m_{2}\right)\left(m_{2}-d_{2}\right)+d_{1}=m_{1}+\eta$ for some $\eta \geqslant 0$. Compute

$$
\frac{y^{\prime}}{y}-\frac{c_{2}}{m_{2}} \frac{z^{\prime}}{z}=\frac{m_{1} x}{x+y}-d_{1}-c_{2}+\frac{c_{2}}{m_{2}} d_{2}=\left(m_{1}-\frac{m_{1} y}{x+y}-d_{1}\right)-\frac{c_{2}}{m_{2}}\left(m_{2}-d_{2}\right)=-\frac{m_{1} y}{x+y}-\eta<0 .
$$

Hence

$$
y(t) \leqslant C(z(t))^{c_{2} / m_{2}} \exp \left(\int_{t_{0}}^{t}-\frac{m_{1} y(s)}{x(s)+y(s)} \mathrm{d} s\right), \quad t \geqslant 0
$$

for some constant $C>0$. We claim that $\lim _{t \rightarrow \infty} y(t)=0$. Assume otherwise, then the positivity and boundedness of the solution together imply the existence of a positive constant $M$, such that $\left|y^{\prime}(t)\right|<M$ for all $t \geqslant 0$, This in turn implies the uniform continuity of $y(t)$ and hence

$$
\int_{0}^{\infty} y(t) \mathrm{d} t=+\infty
$$

From Lemma 2.2, we see that there is a positive constant $L$, such that $x(t)+y(t)<L$ for $t \geqslant 0$. We thus have

$$
\int_{0}^{t} \frac{m_{1} y(s)}{x(s)+y(s)} \mathrm{d} s \geqslant \frac{m_{1}}{L} \int_{0}^{t} y(s) \mathrm{d} s .
$$

This shows that $\lim _{t \rightarrow \infty} \exp \left(\int_{0}^{t}-\left(m_{1} y(s) / x(s)+y(s)\right) \mathrm{d} s\right)=0$ and hence $\lim _{t \rightarrow \infty} y(t)=0$.

If $\lim _{t \rightarrow \infty} z(t)=\eta>0$ then for small $\epsilon>0$ there exists $t_{0}>0$ such that $0<y(t)<\epsilon$ and $\eta-\epsilon<z(t)<\eta+\epsilon$ for $t \geqslant t_{0}$. From (2.3) it follows that

$$
\begin{aligned}
z(t) & =z\left(t_{0}\right) \exp \left(\int_{t_{p}}^{t}\left(\frac{m_{2} y(s)}{y(s)+z(s)}-d_{2}\right) \mathrm{d} s\right) \\
& \leqslant z\left(t_{0}\right) \exp \left(\int_{t_{0}}^{t}\left(\frac{m_{2} \epsilon}{\eta-\epsilon}-d_{2}\right) \mathrm{d} s\right) \rightarrow 0
\end{aligned}
$$

as $t \rightarrow \infty$. If $\lim _{t \rightarrow \infty} z(t)$ does not exist then $\bar{z}=\lim \sup _{t \rightarrow \infty} z(t)>0$. Then there exists $\left\{t_{n}\right\} \uparrow \infty$ such that $z^{\prime}\left(t_{n}\right)=0$ and $z\left(t_{n}\right) \rightarrow \bar{z}>0$ as $n \rightarrow \infty$. From (2.3) we have

$$
\frac{m_{2} y\left(t_{n}\right)}{y\left(t_{n}\right)+z\left(t_{n}\right)}=d_{2} \text {. }
$$

Letting $n \rightarrow \infty$ in (2.11) yields a desired contradiction.

Our first theorem of this section gives conditions for the total extinction of all the three species and conditions of the extinction of both middle and top predators (but not the prey species $x$ ). The first part of the theorem states that if the middle predator is a high capacity and aggressive consumer (characterized by large values of $c_{1}$ ) and there is a shortage of prey to begin with, then all three species will go extinct. The second part of the theorem suggests that if middle predator is a low capacity consumer, then prey species will persist. If we are to think $x$ as a plant species, $y$ as a pest species and $z$ as a species used to control the pest, then these conditions provide scenarios when such biological control may or may not be successful (success is characterized by $\left.\lim _{t \rightarrow \infty}(x(t), y(t), z(t))=(1,0,0)\right)$. 
Theorem 2.1. Assume that $m_{2}>d_{2}$ and $0<A \leqslant 1$. If $c_{1}>1+d_{1}+c_{2}$ and $x(0) / y(0)<\left(c_{1}-(1+\right.$ $\left.\left.d_{1}+c_{2}\right)\right) /\left(1+d_{1}+c_{2}\right) \equiv \delta$, then $\lim _{t \rightarrow \infty}(x(t), y(t), z(t))=(0,0,0)$. If $c_{1}<1$, then $\lim _{t \rightarrow \infty}(x(t)$, $y(t), z(t))=(1,0,0)$.

Proof. From Lemma 2.4, we have $\lim _{t \rightarrow \infty} y(t)=0$ and $\lim _{t \rightarrow \infty} z(t)=0$.

Assume first that $c_{1}>1+d_{1}+c_{2}$ and $x(0) / y(0)<\delta$. We claim that $x(t) / y(t)<\delta$ for all $t>0$. Otherwise, there is a $t_{1}>0$ such that $x(t) / y(t)<\delta$ for $t \in\left[0, t_{1}\right]$ and $x\left(t_{1}\right) / y\left(t_{1}\right)=\delta$. Thus for $t \in\left[0, t_{1}\right]$, we have

$$
x^{\prime}(t)<x-\frac{c_{1} x}{1+(x / y)} \leqslant\left(1-c_{1} /(1+\delta)\right) x
$$

and

$$
y^{\prime}>\left(-d_{1}-c_{2}\right) y
$$

which yield

$$
x(t)<x(0) \exp \left(\left(1-c_{1} /(1+\delta)\right) t\right)=x(0) \exp \left(-\left(d_{1}+c_{2}\right) t\right)
$$

and

$$
y(t)>y(0) \exp \left(-\left(d_{1}+c_{2}\right) t\right)
$$

respectively. Therefore, for $t \in\left[0, t_{1}\right]$, we have

$$
\frac{x(t)}{y(t)}<\frac{\left[x(0) \exp \left(\left(1-c_{1} /(1+\delta)\right) t\right)\right]}{\left[y(0) \exp \left(-\left(d_{1}+c_{2}\right) t\right)\right]}=\frac{x(0)}{y(0)}<\delta .
$$

This proves our claim. Clearly, the proof of this claim also shows that for all $t>0$, we have $x(t)<x(0) \exp \left(-\left(d_{1}+c_{2}\right) t\right) \rightarrow 0$ as $t \rightarrow \infty$. This proves that $\lim _{t \rightarrow \infty}(x(t), y(t), z(t))=(0,0,0)$.

Assume now that $c_{1}<1$. Then we have $x^{\prime}(t)>x-x^{2}-c_{1} x=x\left(1-c_{1}-x\right)$. Simple comparison argument shows that $\lim \inf _{t \rightarrow \infty} x(t) \geqslant 1-c_{1}>0$. Hence, for any $c_{1}>\varepsilon>0$, there is a $t_{2}>0$, such that for $t>t_{2}, y(t)<\left[\left(1-c_{1}\right) / 2\right]\left[\varepsilon /\left(c_{1}-\varepsilon\right)\right]$ and $x(t)>\left(1-c_{1}\right) / 2$. This implies that for $t_{2}>0, c_{1} y /(x+y)<\varepsilon$. Hence

$$
x^{\prime}(t)>x(1-\varepsilon-x) .
$$

Again, simple comparison argument shows that $\lim _{\inf _{t \rightarrow \infty}} x(t) \geqslant 1-\varepsilon$. Letting $\varepsilon \rightarrow 0$, we have $\lim _{t \rightarrow \infty}(x(t), y(t), z(t))=(1,0,0)$.

Lemma 2.5. If $m_{2}>d_{2}, A>1$ and $c_{1} \geqslant A /(A-1)$, then $\lim _{t \rightarrow \infty} z(t)=0$.

Proof. The assumptions $A>1$ and $c_{1} \geqslant A /(A-1)$ imply $\left(1 / c_{1}\right)-1+(1 / A)=-\eta \leqslant 0$. One has

$$
\begin{aligned}
\frac{m_{1}}{c_{1}} \frac{x^{\prime}}{x}-\frac{y^{\prime}}{y}+\frac{c_{2}}{m_{2}} \frac{z^{\prime}}{z} & =\frac{m_{1}}{c_{1}}(1-x)-m_{1}+d_{1}+c_{2}-\frac{c_{2}}{m_{2}} d_{2} \\
& =\frac{m_{1}}{c_{1}}-\frac{m_{1}}{c_{1}} x-m_{1}+\frac{m_{1}}{A} \\
& \leqslant-\frac{m_{1}}{c_{1}} x<0 .
\end{aligned}
$$


We have

$$
(x(t))^{m_{1} / c_{1}}(z(t))^{c_{2} / m_{2}} \leqslant C(y(t)) \exp \left(-\int_{0}^{t} \frac{m_{1}}{c_{1}} x(s) \mathrm{d} s\right)
$$

for some $C>0$. We claim

$$
x(t) z(t) \rightarrow 0 \quad \text { as } \quad t \rightarrow \infty .
$$

If not, then $\lim _{t \rightarrow \infty} x(t) \neq 0$ (and $\lim _{t \rightarrow \infty} z(t) \neq 0$ ). A similar argument for the proof of $\int_{0}^{\infty} y(t) \mathrm{d} t=$ $+\infty$ in the previous lemma yields that

$$
\int_{0}^{\infty} x(t) \mathrm{d} t=+\infty
$$

which implies (2.12), proving the claim.

Next, we claim that $\lim _{t \rightarrow \infty} z(t)=0$. If not, then either $\lim _{t \rightarrow \infty} z(t)$ exists with positive limit or it does not exist. Assume first the former case. By (2.12), $\lim _{t \rightarrow \infty} z(t)=\bar{z}>0$ implies $\lim _{t \rightarrow \infty} x(t)=0$. Using the same arguments in Lemma 2.4, we can show that $\lim _{t \rightarrow \infty} y(t)=0$ which implies $\lim _{t \rightarrow \infty} z(t)=0$ and this is a contradiction. Assume now the latter case, we have $\lim _{\sup _{t \rightarrow \infty}} z(t)=$ $\bar{z}>0$. There exists $\left\{t_{n}\right\} \uparrow \infty, z^{\prime}\left(t_{n}\right)=0$ and $z\left(t_{n}\right) \rightarrow \bar{z}$ as $n \rightarrow \infty$. We thus have

$$
0=z^{\prime}\left(t_{n}\right)=z\left(t_{n}\right)\left[\frac{m_{2} y\left(t_{n}\right)}{y\left(t_{n}\right)+z\left(t_{n}\right)}-d_{2}\right]
$$

and

$$
y\left(t_{n}\right) \rightarrow \bar{y}=\frac{d_{2} \bar{z}}{m_{2}-d_{2}}>0 .
$$

From (2.12), we have $x\left(t_{n}\right) \rightarrow 0$ as $n \rightarrow \infty$. Hence $P \equiv(0, \bar{y}, \bar{z}) \in \Omega$, the $\omega$-limit set of the trajectory $\{(x(t), y(t), z(t)): t \geqslant 0\}$. By the invariance of $\omega$-limit set, it follows that the backward trajectory of $P$ is contained in $\Omega$. We claim that the backward trajectory $\{(\bar{x}(t), \bar{y}(t), \bar{z}(t)): t \leqslant 0\}$ through $P$ is unbounded. Obviously $\bar{x}(t) \equiv 0$. Let $\tau=-t$, then

$$
\frac{\mathrm{d} y}{\mathrm{~d} \tau}=d_{1} y+\frac{c_{2} y z}{y+z} \geqslant d_{1} y
$$

and

$$
y(\tau) \geqslant y(0) \exp \left(d_{1} \tau\right) \rightarrow \infty \quad \text { as } \quad \tau \rightarrow+\infty .
$$

This contradicts to the boundedness of the solution.

Combining Lemmas 2.3-2.5 we have the following intuitive and sharp extinction results of top predator.

Theorem 2.2. If the interior equilibrium $E_{c}$ does not exist, then the top predator of model (2.1)-(2.3) will die out. Specifically, if one of the following three conditions holds, (i) $m_{2} \leqslant d_{2}$; (ii) $m_{2}>d_{2}, 0<A \leqslant 1$; (iii) $m_{2}>d_{2}, A>1$ and $c_{1} \geqslant A /(A-1)$, then $\lim _{t \rightarrow \infty} z(t)=0$. 
Let $c_{2}=0.5, m_{2}=2, d_{1}=1, d_{2}=1$ and let $c_{1}, m_{1}$ be parameters. Fig. 1 illustrates the parameter ranges for various extinction scenarios.

Our next theorem gives conditions for the origin as a global attractor for system (2.1)-(2.3). Naturally, $E_{c}$ does not exist in such scenario.

Theorem 2.3. If $c_{1}>1$, and $m_{1} \geqslant\left(c_{1} / c_{1}-1\right)\left(d_{1}+c_{2}\right)$, then the origin is globally attractive. That is, $\lim _{t \rightarrow \infty}(x(t), y(t), z(t))=(0,0,0)$.

Proof. Due to the domino effect of the simple food chain, we see that it is sufficient to show that $\lim _{t \rightarrow \infty} x(t)=0$. Let $U(t)=x(t) /(y(t))^{\alpha}$, where $\alpha$ will be determined later. We have

$$
U^{\prime}(t)=\frac{x}{y^{\alpha}}\left[1+\alpha\left(d_{1}+c_{2}\right)-x-\frac{\alpha m_{1} x+c_{1} y}{x+y}-\frac{\alpha c_{2} y}{y+z}\right] .
$$

Let

$$
\beta_{1}=1+\alpha\left(d_{1}+c_{2}-m_{1}\right), \quad \text { and } \quad \beta_{2}=1+\alpha\left(d_{1}+c_{2}\right)-c_{1} .
$$



Fig. 1. Here, $c_{2}=0.5, m_{2}=2, d_{1}=1, d_{2}=1$ and let $c_{1}, m_{1}$ be parameters. In $\Delta_{1}=\left\{\left(c_{1}, m_{1}\right) \mid c_{1}>1 ; m_{1} \geqslant\right.$ $\left.c_{1}\left(d_{1}+c_{2}\right) /\left(c_{1}-1\right)\right\}$, the origin attracts all positive solutions. In $\Delta_{2}=\left\{\left(c_{1}, m_{1}\right) \mid c_{1} \in(0,1) ; m_{1} \leqslant d_{1}+c_{2}\left(m_{2}-d_{2}\right) / m_{2}\right\}$, $(1,0,0)$ attracts all positive solutions. In $\Delta_{3}=\left\{\left(c_{1}, m_{1}\right) \mid d_{1}+c_{2}\left(m_{2}-d_{2}\right) / m_{2}<m_{1}<\left(c_{1} /\left(c_{1}-1\right)\right)\left(d_{1}+c_{2}\left(m_{2}-d_{2}\right) /\right.\right.$ $\left.\left.m_{2}\right)\right\}, E_{c}$ exists. 
Then (2.13) can be rewritten as

$$
U^{\prime}(t)=U\left[\frac{\beta_{1} x+\beta_{2} y}{x+y}-x-\frac{\alpha c_{2} y}{y+z}\right] .
$$

$c_{1}>1$, and $m_{1} \geqslant\left(c_{1} / c_{1}-1\right)\left(d_{1}+c_{2}\right)$ imply the existence of $\alpha$ such that

$$
\frac{1}{m_{1}-d_{1}-c_{2}} \leqslant \frac{c_{1}}{m_{1}} \leqslant \alpha \leqslant \frac{c_{1}-1}{d_{1}+c_{2}} .
$$

For such $\alpha, \beta_{1}$ and $\beta_{2}$ are negative. Hence, we have $U^{\prime}(t)<-x(t) U(t)$ which yields

$$
U(t) \leqslant U(0) \exp \left(-\int_{0}^{t} x(s) \mathrm{d} s\right) .
$$

Similar argument as the proof of $\lim _{t \rightarrow \infty} y(t)=0$ in Lemma 2.4 shows that $\lim _{t \rightarrow \infty} x(t)=0$, proving the theorem.

\section{Stability of $E_{c}$}

In this section, we assume that $E_{c}$ exists and study its local stability. This will yield some analytic (explicit) and computational (implicit) conditions for both stable and oscillatory coexistence of all three species.

The variational matrix of (2.1)-(2.3) at $E_{c}$ is given by

$$
M=\left[\begin{array}{ccc}
m_{11} & m_{12} & 0 \\
m_{21} & m_{22} & m_{23} \\
0 & m_{32} & m_{33}
\end{array}\right]_{(x, y, z)=\left(x_{c}, y_{c}, z_{c}\right)},
$$

where

$$
\begin{aligned}
& m_{11}=x_{c}\left[-1+\frac{c_{1} y_{c}}{\left(x_{c}+y_{c}\right)^{2}}\right], \\
& m_{22}=y_{c}\left[-\frac{m_{1} x_{c}}{\left(x_{c}+y_{c}\right)^{2}}+\frac{c_{2} z_{c}}{\left(z_{c}+y_{c}\right)^{2}}\right], \\
& m_{33}=-\frac{m_{2} y_{c} z_{c}}{\left(y_{c}+z_{c}\right)^{2}}, \\
& m_{12}=-\frac{c_{1} x_{c}^{2}}{\left(x_{c}+y_{c}\right)^{2}}, \quad m_{21}=\frac{m_{1} y_{c}^{2}}{\left(x_{c}+y_{c}\right)^{2}}, \quad m_{23}=-\frac{c_{2} y_{c}^{2}}{\left(y_{c}+z_{c}\right)^{2}}, \quad m_{32}=\frac{m_{2} z_{c}^{2}}{\left(y_{c}+z_{c}\right)^{2}} .
\end{aligned}
$$

The characteristic polynomial of $M$ is

$$
f(\lambda)=\operatorname{det}(M-\lambda I)=\left(m_{11}-\lambda\right)\left(m_{22}-\lambda\right)\left(m_{33}-\lambda\right)-m_{12} m_{21}\left(m_{33}-\lambda\right)-m_{23} m_{32}\left(m_{11}-\lambda\right) .
$$


Then the roots $\lambda$ of $f(\lambda)=0$ satisfy

$$
\lambda^{3}+A_{1} \lambda^{2}+A_{2} \lambda+A_{3}=0,
$$

where

$$
\begin{aligned}
& A_{1}=-m_{11}-m_{22}-m_{33} \\
& A_{2}=m_{22} m_{33}+m_{11} m_{22}+m_{11} m_{33}-m_{12} m_{21}-m_{23} m_{32}, \\
& A_{3}=m_{12} m_{21} m_{33}+m_{11} m_{23} m_{32}-m_{11} m_{22} m_{33} .
\end{aligned}
$$

Straightforward computation shows that

$$
A_{3}=-\operatorname{det} M=m_{1} m_{2} x_{c}^{2} y_{c}^{2} z_{c} /\left[\left(y_{c}+z_{c}\right)\left(x_{c}+y_{c}\right)\right]^{2}>0 .
$$

From Roth-Hurwitz criterion, $E_{c}$ is local asymptotically stable if and only if $A_{1}>0, A_{3}>0$ and $A_{1} A_{2}>A_{3}$.

In the following proposition, a sufficient condition is given for the local stability of $E_{c}$.

Proposition 3.1. If $m_{11}<0$ and $m_{22}<0$ then $E_{c}$ is locally asymptotically stable.

Proof. From the signs of those defined $m_{i j}, i, j=1,2,3$, it is easy to verify $A_{1}>0, A_{2}>0$ and $A_{3}>0$. Expand $A_{1} A_{2}$ and calculate $A_{1} A_{2}-A_{3}$, then

$$
\begin{aligned}
A_{1} A_{2}-A_{3}= & -\left(m_{11}\right)^{2} m_{22}-\left(m_{11}\right)^{2} m_{33}+m_{11} m_{12} m_{21}-\left(m_{22}\right)^{2} m_{33}-m_{11}\left(m_{22}\right)^{2}-2 m_{11} m_{22} m_{33} \\
& +m_{22} m_{12} m_{21}+m_{23} m_{32} m_{22}-m_{22}\left(m_{33}\right)^{2}-m_{11}\left(m_{33}\right)^{2}+m_{23} m_{32} m_{33}>0 .
\end{aligned}
$$

Hence $E_{c}$ is local asymptotically stable.

We can express the conditions $m_{11}<0, m_{22}<0$ in terms of $c_{1}, c_{2}$. From (3.1), (2.4), (2.7), and (2.9), we have

$$
m_{11}=x_{c}\left(-1+\frac{c_{1}(A-1) x_{c}}{A^{2} x_{c}^{2}}\right)<0
$$

if and only if $c_{1}(A-1)<A^{2} x_{c}=A\left(1+\left(1-c_{1}\right)(A-1)\right)$. Clearly, $0<c_{1} \leqslant 1$ implies $m_{11}<0$. If $c_{1}>1$, then $m_{11}<0$ if and only if $1<A<\left(c_{1} /\left(c_{1}-1\right)\right)^{1 / 2}$.

From (3.2), (2.4), (2.7), (2.8), and (2.9), we have

$$
\begin{aligned}
m_{22}<0 & \Longleftrightarrow \frac{c_{2} z_{c}}{\left(z_{c}+y_{c}\right)^{2}}<\frac{m_{1} x_{c}}{\left(x_{c}+y_{c}\right)^{2}} \\
& \Longleftrightarrow c_{2} \frac{\left(m_{2} / d_{2}\right)-1}{\left(m_{2} / d_{2}\right)^{2}}<\frac{m_{1}(A-1)}{A^{2}} \\
& =m_{1}\left(\frac{1}{A}-\frac{1}{A^{2}}\right)=\left(c_{2} \frac{m_{2}-d_{2}}{m_{2}}+d_{1}\right)-\frac{1}{m_{1}}\left(c_{2} \frac{m_{2}-d_{2}}{m_{2}}+d_{1}\right)^{2} \\
& \Longleftrightarrow 0<g\left(c_{2}\right),
\end{aligned}
$$

where

$$
g\left(c_{2}\right)=-\frac{1}{m_{1}}\left(c_{2} \frac{m_{2}-d_{2}}{m_{2}}+d_{1}\right)^{2}+c_{2}\left(\frac{m_{2}-d_{2}}{m_{2}}\right)+d_{1}-c_{2} \frac{\left(m_{2} / d_{2}\right)-1}{\left(m_{2} / d_{2}\right)^{2}} .
$$

Since $g(0)>0$ and $g\left(\frac{m_{1}-d_{1}}{\left(m_{2}-d_{2}\right) / m_{2}}\right)<0$, there exist $c_{2}^{*}>0$ such that $g\left(c_{2}^{*}\right)=0$. 




Fig. 2. The parameter region for the the asymptotical stability of $E_{c}$ given by Proposition 3.1 , when $m_{1}=1.5, m_{2}=2$, $d_{1}=1, d_{2}=1$. The region, denoted by $\mathbf{R}$, is bounded by the two axes and the two curves defined by $c_{2}=c_{2}^{*}$ and $A=\left(c_{1} /\left(c_{1}-1\right)\right)^{1 / 2}$.

Then

$$
0<c_{2}<c_{2}^{*} \Longleftrightarrow m_{22}<0 .
$$

To discuss the instability of $E_{c}$ we consider the possibility of choosing $c_{1}, c_{2}$ satisfying $A_{1}<0$. By (3.1)-(3.3), we have (Fig. 2)

$$
A_{1}=I_{1}+I_{2},
$$

where

$$
I_{1}=x_{c}+\left(m_{1}-c_{1}\right) \frac{(A-1)}{A^{2}}
$$

and

$$
I_{2}=\left(m_{2}-c_{2}\right) \frac{\left(m_{2} / d_{2}\right)-1}{\left(m_{2} / d_{2}\right)^{2}} .
$$

Observe that $A=1$ if and only if $c_{2}=m_{2}\left(m_{1}-d_{1}\right) /\left(m_{2}-d_{2}\right)$. Hence we assume

$$
m_{2}<m_{2}\left(m_{1}-d_{1}\right) /\left(m_{2}-d_{2}\right) \text {, i.e. } \quad m_{2}-d_{2}<m_{1}-d_{1}
$$


and consider the case

$$
m_{2}<c_{2}<\frac{m_{2}\left(m_{1}-d_{1}\right)}{m_{2}-d_{2}},
$$

then it follows that $I_{2}<0$.

Notice that

$$
I_{1}=\frac{1}{A}\left(1+\left(1-c_{1}\right)(A-1)\right)+\left(m_{1}-c_{1}\right) \frac{A-1}{A^{2}} .
$$

If $A \approx 1$ then $c_{2} \approx m_{2}\left(m_{1}-d_{1}\right) /\left(m_{2}-d_{2}\right), I_{1} \approx 1$ and

$$
\begin{aligned}
I_{2} & \approx\left(m_{2}-m_{2}\left(m_{1}-d_{1}\right) /\left(m_{2}-d_{2}\right)\right) \frac{\left(\left(m_{2} / d_{2}\right)-1\right)}{\left(m_{2} / d_{2}\right)^{2}} \\
& =\left(d_{2} / m_{2}\right)\left[\left(m_{2}-d_{2}\right)-\left(m_{1}-d_{1}\right)\right]<0 .
\end{aligned}
$$

If we choose $m_{i}, d_{i}, i=1,2$ such that

$$
1+\left[\left(m_{2}-d_{2}\right)-\left(m_{1}-d_{1}\right)\right] d_{2} / m_{2}<0
$$



Fig. 3. The parameter region for the the asymptotical stability of $E_{c}$, when $m_{1}=10, m_{2}=2, d_{1}=1, d_{2}=1$. The dot line filled region, denoted by $\mathbf{R}_{2}$, is bounded by the two axes and the two curves defined by $A=c_{1} /\left(c_{1}-1\right)$ and $A_{1} A_{2}=A_{3}$. $A_{1}=0$ divides the figure into two parts, on the top and right side of it, $A_{1}<0$. So, even though $A_{1} A_{2}>A_{3}$ on the top of the existence region of $E_{c}, E_{c}$ remains unstable. 
or

$$
m_{2} / d_{2}<\left(m_{1}-d_{1}\right)-\left(m_{2}-d_{2}\right),
$$

then $A_{1}<0$ for $c_{2}$ near $m_{2}\left(m_{1}-d_{1}\right) /\left(m_{2}-d_{2}\right)$. Thus we have the following proposition.

Proposition 3.2. Let (3.5) and (3.6) hold. Then $E_{c}$ is unstable for $m_{2}<c_{2}<m_{2}\left(m_{1}-d_{1}\right) /\left(m_{2}-d_{2}\right)$ and $c_{2}$ is near $m_{2}\left(m_{1}-d_{1}\right) /\left(m_{2}-d_{2}\right)$.

There are two possible ways for $E_{c}$ to change from stable to unstable: (1) at least one of its eigenvalues change from negative to positive, and hence at the transition stage, at least one of the eigenvalue assumes the value zero; (2) the real part of a pair of complex-conjugates eigenvalues changes sign. Since when $E_{c}$ exist, we always have $-\operatorname{det} M=-\lambda_{1} \lambda_{2} \lambda_{3}>0$, we see only the second way is actually taken. In Fig. 3, we choose $m_{1}=10, m_{2}=2, d_{1}=d_{2}=1$ and we plot the regions $\mathbf{R}_{\mathbf{1}}, \mathbf{R}_{\mathbf{2}}, \mathbf{R}_{\mathbf{3}}$ in the $c_{1}-c_{2}$ parameter plane where the equilibrium $E_{c}$ does not exist in $\mathbf{R}_{\mathbf{1}}$; in region $\mathbf{R}_{\mathbf{2}}$, $E_{c}$ is local asymptotically stable with one negative eigenvalue, two complex-conjugates with negative real parts; in region $\mathbf{R}_{\mathbf{3}}, E_{c}$ is a saddle point with one negative eigenvalue and two complex-conjugates with positive real parts. Clearly, Hopf bifurcation occurs if the parameters $c_{1}$, $c_{2}$ cross the boundary of $\mathbf{R}_{\mathbf{2}}$ and $\mathbf{R}_{\mathbf{3}}, \partial \mathbf{R}_{\mathbf{2}} \cap \partial \mathbf{R}_{\mathbf{3}}$.

(a) A periodic solution

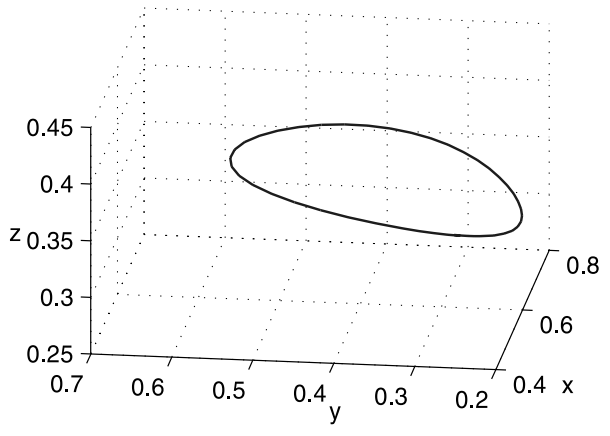

(c)



(b) The periodic solution in t

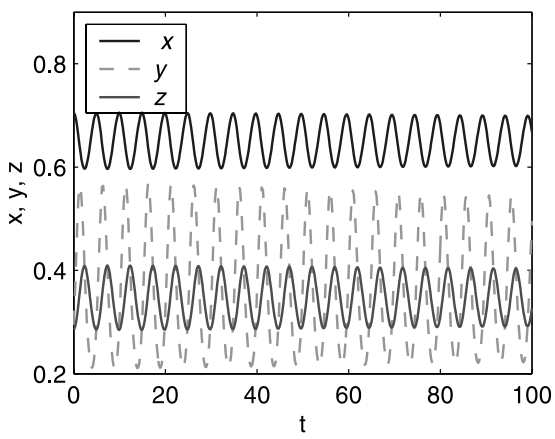

(d)

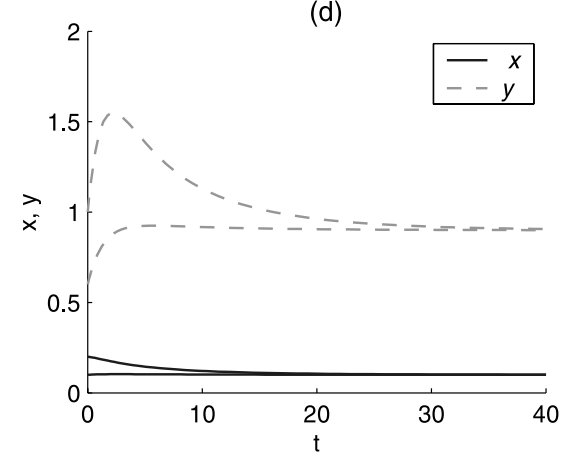

Fig. 4. (a) Depicts a positive periodic solution of system (2.1)-(2.3), where $m_{1}=10, m_{2}=2, d_{1}=1, d_{2}=1, c_{1}=1$, $c_{2}=11, x(0)=0.7046, y(0)=0.2941$ and $z(0)=0.2853$. (b) Depicts the time course of this periodic solution. When we reduce $c_{2}$ from 11 to $10, E_{c}$ becomes asymptotically stable. (c) Depicts the time course of a solution tending to $E_{c}$. (d) Shows that without top predator, species $x$ and $y$ coexist in the form of a stable steady state. 
In Fig. 4(a), we show a positive periodic solution of system (2.1)-(2.3), where $m_{1}=10, m_{2}=2$, $d_{1}=1, d_{2}=1, c_{1}=1, c_{2}=11, x(0)=0.7046, y(0)=0.2941$ and $z(0)=0.2853$. For this set of parameters, Fig. 3 indicates that system (2.1)-(2.3) has a unstable positive steady state $E_{c}$. Extensive simulation study of this example shows that the solutions are very sensitive to the initial populations and the system appears to have a chaotic attractor resembles a closed band. Most of its solutions approaches this band along a tea cup shape surface (see Fig. 8(b) in Section 5).

A plausible biological implication of our findings in this section (see Fig. 3 ) is that given a predator $(y)$ prey $(x)$ interaction, the successful introduction of a top predator $(z)$ is very sensitive to the its effectiveness in catching the predator $y$, which is measured by the parameter $c_{2}$. If predator $y$ is a voracious one (characterized as having high values of $c_{1}$ ) for prey $x$, then both high (may over prey on $y$ ) and low effective top predators $z$ are doomed. If predator $y$ is not so voracious for prey $x$, then high effective top predators $z$ are doomed while low and medium effective ones may endure. Moreover, low effective top predators likely coexist with species $y$ and thus $x$ in the form of stable equilibria, while medium effective top predators may generate population fluctuations in all the three species.

The implications of this section on biological control is somewhat subtle, we leave this to the discussion section.

\section{Sensitivity to initial conditions}

It is well known that prey-dependent simple food chain models can generate chaotic dynamics [26-30], which indicates that for some parameters, solutions can be very sensitive to initial populations. We shall see that the ratio-dependent simple food chain model (2.1)-(2.3) is also capable of generating these sensitive dynamics.

Theorem 2.2 implies that no complex dynamics is possible for system (2.1)-(2.3) if it has no positive steady state $E_{c}$. Hence, in this section, we do not assume that the interior equilibrium $E_{c}=\left(x_{c}, y_{c}, z_{c}\right)$ does not exists. In the $x y z$ space, in addition to the possible $E_{c}$, there maybe equilibria $(0,0,0),(1,0,0),\left(x^{*}, y^{*}, 0\right)$. From the Eq. (2.3), the equilibrium $\left(x^{*}, y^{*}, 0\right)$ or the periodic orbit $\Gamma$ in $x y$ plane (if $\Gamma$ exists where $\Gamma=\left\{\left(x^{*}(t), y^{*}(t), 0\right): 0 \leqslant t \leqslant T\right\}, T$ is the period) is unstable in $z$-direction (when $z(0)$ is small and $(x(0), y(0), z(0))$ is near to the steady state $\left(x^{*}, y^{*}, 0\right)$ or $\Gamma=\left\{\left(x^{*}(t), y^{*}(t), 0\right): 0 \leqslant t \leqslant T\right\}$, we have $\left.\ln (z(T) / z(0)) \approx \exp \left(\left(m_{2}-d_{2}\right) T\right)>1\right)$. Hence we only need to discuss the possibilities of trajectories tending to $(0,0,0)$ or $(1,0,0)$. First we consider the conditions for the existence of trajectories tending to $(0,0,0)$. To this end, we introduce $u=x / y$ and $v=y / z$ and the change of variables $(x, y, z) \rightarrow(u, y, v)$. Then (2.1)-(2.3) is converted in to following system:

$$
\begin{aligned}
u^{\prime} & =\frac{u}{1+u}(A u+B)-u^{2} y+\frac{c_{2} u}{1+v}, \quad u(0)>0, \\
y^{\prime} & =\left(\frac{m_{1} u}{1+u}-d_{1}\right) y-\frac{c_{2} y}{1+v}, \quad y(0)>0, \\
v^{\prime} & =\left[\left(\frac{m_{1} u}{1+u}-d_{1}\right)-\frac{c_{2}}{v+1}-\left(\frac{m_{2} v}{1+v}-d_{2}\right)\right] v, \quad v(0)>0,
\end{aligned}
$$


where

$$
A=1-\left(m_{1}-d_{1}\right), \quad B=1-c_{1}+d_{1} .
$$

The system (4.1), (4.2) and (4.3) has the following equilibria of the form

$$
\begin{aligned}
& E_{00}=(0,0,0), \\
& E_{01}=(0,0, \hat{v}), \quad \hat{v}>0, \\
& E_{10}=\left(\theta_{1}^{*}, 0,0\right), \quad \theta_{1}^{*}>0, \\
& E_{11}=\left(\theta_{2}^{*}, 0, \tilde{v}\right), \quad \theta_{2}^{*}>0, \tilde{v}>0 .
\end{aligned}
$$

From the domino effect of simple food chain, it is easy to see that

1. $(u(t), y(t), v(t)) \rightarrow E_{00}$ as $t \rightarrow \infty$ if and only if $(x(t), y(t), z(t)) \rightarrow(0,0,0)$ and $x(t) \rightarrow 0$ faster than $y(t) \rightarrow 0, y(t) \rightarrow 0$ faster than $z(t) \rightarrow 0$.

2. $(u(t), y(t), v(t)) \rightarrow E_{01}$ if and only if $(x(t), y(t), z(t)) \rightarrow(0,0,0)$ and $x(t) \rightarrow 0$ faster than $y(t) \rightarrow 0, z(t) \rightarrow 0$ at finite rate as $y(t) \rightarrow 0$.

3. $(u(t), y(t), v(t)) \rightarrow E_{10}$ if and only if $(x(t), y(t), z(t)) \rightarrow(0,0,0)$ and $y(t) \rightarrow 0$ faster than $z(t)$, $x(t) \rightarrow 0$ at finite rate as $y(t) \rightarrow 0$.

4. $(u(t), y(t), v(t)) \rightarrow E_{11}$ if and only if $y(t) \rightarrow 0$ and $x(t) \rightarrow 0, z(t) \rightarrow 0$ at finite rate as $y(t) \rightarrow 0$.

The equilibrium $E_{00}=(0,0,0)$ of (4.1)-(4.3) always exists. The variational matrix at $E_{00}$ is

$$
J\left(E_{00}\right)=\left[\begin{array}{ccc}
B+c_{2} & 0 & 0 \\
0 & -d_{2}-c_{2} & 0 \\
0 & 0 & -d_{1}-c_{2}+d_{2}
\end{array}\right] .
$$

From (4.4), $E_{00}$ is asymptotically stable if and only if

$$
1+d_{1}+c_{2}<c_{1} \text { and } d_{2}-d_{1}<c_{2} \text {. }
$$

For the existence of the equilibrium $E_{01}=(0,0, \hat{v})$, from Eq. (4.3), $\hat{v}>0$ satisfies

$$
d_{2}-d_{1}=\frac{m_{2} \hat{v}+c_{2}}{\hat{v}+1} .
$$

Hence

$E_{01}$ exists if and only if $d_{2}-d_{1}>0$ and $d_{2}-d_{1}$ is between $m_{2}$ and $c_{2}$.

The variational matrix at $E_{01}$ is

$$
J\left(E_{01}\right)=\left[\begin{array}{ccc}
B+\frac{c_{2}}{1+\hat{v}} & 0 & 0 \\
0 & -d_{1}-\frac{c_{2}}{1+\hat{v}} & 0 \\
m_{1} \hat{v} & 0 & \hat{v}\left[\frac{c_{2}}{(\hat{v}+1)^{2}}-\frac{m_{2}}{(1+\hat{v})^{2}}\right]
\end{array}\right] .
$$

$E_{01}$ is asymptotically stable if and only if

$$
1+d_{1}+\frac{c_{2}}{1+\hat{v}}<c_{1} \text { and } \quad c_{2}<m_{2}
$$


This together with (4.7) imply that $E_{01}$ exists and is asymptotically stable if and only if

$$
1+d_{1}+\frac{c_{2}\left(m_{2}+d_{1}-d_{2}\right)}{m_{2}-c_{2}}<c_{1} \quad \text { and } \quad c_{2}<d_{2}-d_{1}<m_{2} .
$$

For the existence of the equilibrium $E_{10}=\left(\theta_{1}^{*}, 0,0\right)$, from Eq. (4.1), $\theta_{1}^{*}$ satisfies

$$
\theta_{1}^{*}=\frac{-\left(B+c_{2}\right)}{A+c_{2}} .
$$

Hence

$$
E_{10} \text { exists if and only if }\left(B+c_{2}\right)\left(A+c_{2}\right)<0 .
$$

The variational matrix at $E_{10}$ is

$$
J\left(E_{10}\right)=\left[\begin{array}{ccc}
\frac{(A-B) u}{(1+u)^{2}} & -u^{2} & -c_{2} u \\
0 & \frac{m_{1} u}{1+u}-d_{1}-c_{2} & 0 \\
0 & 0 & \frac{m_{1} u}{1+u}-d_{1}-c_{2}+d_{2}
\end{array}\right]_{u=\theta_{1}^{*}}
$$

$E_{10}$ is asymptotically stable if and only if

$$
A<B, \quad \frac{m_{1} \theta_{1}^{*}}{1+\theta_{1}^{*}}+d_{2}<d_{1}+c_{2} .
$$

This together with (4.4) and (4.10) imply that $E_{10}$ exists and is asymptotically stable if and only if

$$
m_{1}>1+d_{1}+c_{2}>c_{1}, \quad m_{1}\left(1+d_{2}-c_{1}\right)<c_{1}\left(d_{2}-c_{2}-d_{1}\right) .
$$

For example, $d_{1}=0.6, d_{2}=0.24, m_{1}=3, m_{2}=1, c_{1}=2, c_{2}=0.6$ satisfy the above condition.

For the existence of the equilibrium $E_{11}=\left(\theta_{2}^{*}, 0, \tilde{v}\right),(4.1)$ and (4.3) imply that $\theta_{2}^{*}$ and $\tilde{v}$ satisfy

$$
\frac{B+A \theta_{2}^{*}}{1+\theta_{2}^{*}}+\frac{c_{2}}{1+\tilde{v}}=0,
$$

and

$$
\frac{m_{1} \theta_{2}^{*}}{1+\theta_{2}^{*}}-d_{1}-\frac{c_{2}}{1+\tilde{v}}-\left(\frac{m_{2} \tilde{v}}{\tilde{v}+1}-d_{2}\right)=0 .
$$

The stability analysis of $E_{11}$ is rather complicated and we forgo it here.

Our next result provides another set of conditions for the origin to be a local attractor. Notice that the result is an improvement of the first part of Theorem 2.1 when $m_{1}<c_{1}\left(d_{1}+c_{2}\right) /\left(c_{1}-1\right)$. In addition, the conditions do not exclude the existence of $E_{c}$.

Proposition 4.1. Assume that $c_{1}>1+d_{1}+c_{2}$ and $m_{1}<c_{1}\left(d_{1}+c_{2}\right) /\left(c_{1}-1\right)$. If, in addition,

$$
\frac{x(0)}{y(0)} \leqslant \frac{c_{1}-\left(1+d_{1}+c_{2}\right)}{1+d_{1}+c_{2}-m_{1}} \equiv u_{0},
$$

then $\lim _{t \rightarrow \infty}(x(t), y(t), z(t))=(0,0,0)$. 
Proof. Due to the domino effect of the simple food chain, we need only to show that $\lim _{t \rightarrow \infty} x(t)=$ 0 . Let $u(t)=x(t) / y(t)$. We have

$$
u^{\prime}(t)=u\left[\left(1+d_{1}+c_{2}-m_{1}\right) \frac{u-u_{0}}{1+u}-x-\frac{c_{2} y}{y+z}\right] .
$$

Clearly, for $t>0, u(t) \leqslant u_{0}$ if $u(0) \leqslant u_{0}$. Hence

$$
u(t) \leqslant u(0) \exp \left(-\int_{0}^{t} x(s) \mathrm{d} s\right) .
$$

The rest follows from the argument of Theorem 2.3 that yields $\lim _{t \rightarrow \infty} x(t)=0$.

Next we consider the conditions for the existence of trajectories of (2.1)-(2.3) tending to $E_{1}=(1,0,0)$ as $t \rightarrow \infty$.

Proposition 4.2. Assume that $m_{2}>d_{2}$ and $m_{1}<\min \left\{1+d_{1}, d_{1}+c_{2}-d_{2}\right\}$. Then there are solutions of (2.1)-(2.3) tending to $E_{1}=(1,0,0)$ as $t \rightarrow \infty$.

Proof. Observe that (4.1)-(4.3) can be rewritten as

$$
\begin{aligned}
u^{\prime} & =u^{2}\left[\frac{A u+B}{u(1+u)}+\frac{c_{2}}{u(1+v)}-y\right]=u^{2}[K(u, v)-y] \equiv G_{1}(u, y, v), \quad u(0)>0, \\
y^{\prime} & =y\left[\frac{-m_{1}}{1+u}+\frac{l_{1} v+l_{2}}{1+v}\right] \equiv G_{2}(u, y, v), \quad y(0)>0, \\
v^{\prime} & =v\left[\frac{-m_{1}}{1+u}+\frac{q_{1} v+q_{2}}{1+v}\right] \equiv G_{3}(u, y, v), \quad v(0)>0,
\end{aligned}
$$

where $A=1-m_{1}+d_{1}, B=1-c_{1}+d_{1}, l_{1}=m_{1}-d_{1}, l_{2}=m_{1}-d_{1}-c_{2}, q_{1}=m_{1}-d_{1}+d_{2}-m_{2}$, $q_{2}=m_{1}-d_{1}+d_{2}-c_{2}$.

Clearly, $A>0>q_{2}>l_{2}$. Hence there is a $v_{0}>0$ such that $G_{2}(u, y, v)<0$ and $G_{3}(u, y, v)<0$ for all $(u, y, v) \in[0, \infty) \times[0, \infty) \times\left[0, v_{0}\right]$. Let $u_{0}=\max \{-B / A, 0\} \geqslant 0$ and

$$
\Omega \equiv\left\{(u, y, v) \in \mathbf{R}_{+}^{3} \mid u \in\left[u_{0}, \infty\right), v \in\left[0, v_{0}\right], y \in\left[0, K\left(u_{0}, v_{0}\right)\right]\right\} .
$$

Then $\Omega$ is positively invariant. Since there is no equilibrium in $\Omega$, the monotonicity of the components of the solution in $\Omega$ implies that $\lim _{t \rightarrow \infty}(u(t), y(t), v(t))=(+\infty, 0,0)$. Therefore, if $x(0) \geqslant u_{0} y(0), y(0) \leqslant v_{0} z(0)$ and $y(0)<K\left(u_{0}, v_{0}\right)$, then $\lim _{t \rightarrow \infty} x(t) / y(t)=+\infty$. This shows that for any $\varepsilon>0$, there is a $T_{\varepsilon}>0$ such that for $t \geqslant T_{\varepsilon}$, we have

$$
\frac{c_{1} y(t)}{x(t)+y(t)}=\frac{c_{1}}{1+x(t) / y(t)}<\varepsilon .
$$

From (2.1), we have $x^{\prime}(t)>x(t)(1-\varepsilon-x(t))$ for all $t \geqslant T_{\varepsilon}$. Standard argument shows that $\lim _{t \rightarrow \infty} x(t)=1$, proving the proposition.

To obtain additional results on the conditions for the existence of trajectories of (2.1)-(2.3) tending to $E_{1}=(1,0,0)$ as $t \rightarrow \infty$, we let $v=y / z$ and consider the transformation $(x, y, z) \rightarrow$ $(x, y, v)$. Then $(2.1)-(2.3)$ is converted into the following system: 


$$
\begin{aligned}
x^{\prime} & =x(1-x)-\frac{c_{1} x y}{x+y}, \quad x(0)>0, \\
y^{\prime} & =\left(\frac{m_{1} x}{x+y}-d_{1}-\frac{c_{2}}{v+1}\right) y, \quad y(0)>0, \\
v^{\prime} & =v\left[\left(\frac{m_{1} x}{x+y}-d_{1}-\frac{c_{2}}{v+1}\right)-\left(\frac{m_{2} v}{v+1}-d_{2}\right)\right], \quad v(0)>0 .
\end{aligned}
$$

The system (4.12) has the following equilibria of the form

$$
\begin{aligned}
& E_{100}=(1,0,0), \\
& E_{101}=(1,0, \hat{v}), \quad \hat{v}>0 .
\end{aligned}
$$

$E_{100}$ always exists. From the third equation of (4.12), $\hat{v}$ satisfies

$$
m_{1}-d_{1}+d_{2}=\frac{m_{2} \hat{v}+c_{2}}{\hat{v}+1} .
$$

Hence $E_{101}$ exists if and only if $m_{1}-d_{1}+d_{2}$ is between $m_{2}$ and $c_{2}$.

The variational matrix of (4.12) at $E_{100}$ is

$$
J\left(E_{100}\right)=\left[\begin{array}{ccc}
-1 & -c_{1} & 0 \\
0 & m_{1}-d_{1}-c_{2} & 0 \\
0 & 0 & m_{1}-d_{1}-c_{2}+d_{2}
\end{array}\right] .
$$

Hence $E_{100}$ is asymptotically stable if and only if

$$
m_{1}+d_{2}<d_{1}+c_{2} \text {. }
$$

The variational matrix of (4.12) at $E_{101}$ is

$$
J\left(E_{101}\right)=\left[\begin{array}{ccc}
-1 & -c_{1} & 0 \\
0 & m_{1}-d_{1}-c_{2} /(\hat{v}+1) & 0 \\
0 & -m_{1} \hat{v} & \hat{v}\left(c_{2}-m_{2}\right) /(\hat{v}+1)^{2}
\end{array}\right] .
$$

Hence $E_{101}$ is asymptotically stable if and only if

$$
m_{1}<d_{1}+\frac{c_{2}}{\hat{v}+1} \quad \text { and } \quad c_{2}<m_{2}
$$

From (4.13) and (4.15) can be rewritten as

$$
\left(m_{2}-d_{2}\right) c_{2}>\left(m_{1}-d_{1}\right) m_{2} \quad \text { and } \quad c_{2}<m_{2}
$$

We summarize some of the key findings of this section into the following theorem.

Theorem 4.1. If at least one of the following four condition hold

(1) $1+d_{1}+c_{2}<c_{1}$ and $d_{2}-d_{1}<c_{2}$;

(2) $1+d_{1}+\left(c_{2}\left(m_{2}+d_{1}-d_{2}\right) / m_{2}-c_{2}\right)<c_{1}$ and $c_{2}<d_{2}-d_{1}<m_{2}$;

(3) $m_{1}>1+d_{1}+c_{2}>c_{1}, m_{1}\left(1+d_{2}-c_{1}\right)<c_{1}\left(d_{2}-c_{2}-d_{1}\right)$;

(4) $c_{1}>1+d_{1}+c_{2}$ and $m_{1}<c_{1}\left(d_{1}+c_{2}\right) /\left(c_{1}-1\right)$. 
Then there are positive solutions tend to the origin. If at least one of the following three conditions hold

(i) $m_{1}+d_{2}<d_{1}+c_{2}$;

(ii) $\left(m_{2}-d_{2}\right) c_{2}>\left(m_{1}-d_{1}\right) m_{2}$ and $c_{2}<m_{2}$;

(iii) $m_{2}>d_{2}$ and $m_{1}<\min \left\{1+d_{1}, d_{1}+c_{2}-d_{2}\right\}$.

Then there are positive solutions tend to the predator-free steady state $(1,0,0)$.

It should be stressed here that the scenarios of having positive solutions tend to the origin or the pest free steady state $(1,0,0)$ can coexist with each other and the existence of positive attractors such as stable $E_{c}$, a limit cycle, or other more complex attractors. The next two figures illustrate two of such scenarios.

In Fig. 5, we let $m_{1}=1.5, m_{2}=2, d_{1}=d_{2}=1, c_{2}=0.5$ and $c_{1}=4$. Then (4.5) hold and hence $E_{00}$ is asymptotically stable. $E_{c}=\left(x_{c}, y_{c}, z_{c}\right)=(0.3333,0.0667,0.0667)$ is unstable. The solution with initial population $(x(0), y(0), z(0))=p=(0.3433,0.0467,0.0767)$ appears to approaching a

(a)



(b)

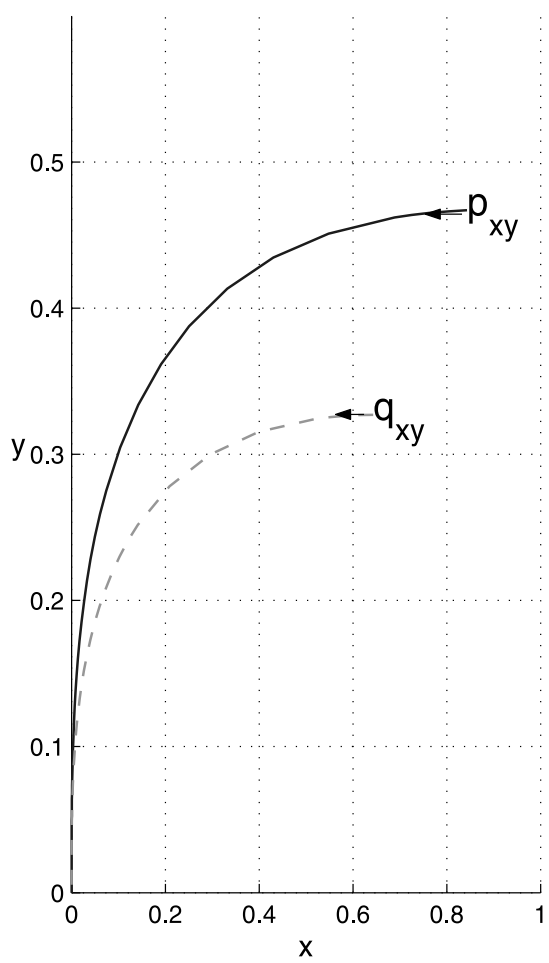

Fig. 5. In (a), two solutions of system (2.1)-(2.3): one tends to a heteroclinic cycle (starting at $p=(0.3433,0.0467$, $0.0767)$ ), another (starting at $q=(0.3433,0.127,0.367)$ ) tends to the origin. Here, $m_{1}=1.5, m_{2}=2, d_{1}=d_{2}=1$, $c_{2}=0.5$ and $c_{1}=4$ and $E_{c}=\left(x_{c}, y_{c}, z_{c}\right)=(0.3333,0.0667,0.0667)$. Then (4.5) hold and hence $E_{00}$ is asymptotically stable. When the top predator is absent, predator $y$ drives prey $x$ to extinction and itself dies out. Here $p_{x y}=$ $(0.3433,0.0467,0)$ and $q_{x y}=(0.3433,0.1270,0)$. 
heteroclinic cycle (in practice, this will be equivalent to the extinction of all the three species). However if we choose the initial condition $(x(0), y(0), z(0))=q=(0.3433,0.1270,0.3670)$, then all species go to extinction (Fig. 5(a)). Therefore solutions are sensitive to initial conditions and the system has multiple attractors. It should be pointed out, with this set of parameters, when the top predator is absent, predator $y$ drives prey $x$ to extinction and itself dies out (see Fig. 5(b)). Thus, for this example, biological control is doomed in a practical sense. This is a scenario where the pest $y$ is very voracious, with or without this particular control agent $z$, it is going to drive the prey $x$ to extinction.

If we choose $m_{1}=1.4, m_{2}=10 / 9, c_{1}=2, c_{2}=1.7, d_{1}=d_{2}=1$ then $E_{c}$ exists and (4.14) holds. Hence $E_{100}$ is asymptotically stable. Our simulation (see Fig. 6(a)) shows $E_{c}=(0.6714,0.1320$, 0.0147 ) is asymptotically stable. As predicted by Theorem 4.1, there are solutions (see Fig. 6(d)) converge to $(1,0,0)$. In this case, when the top predator is absent, predator $y$ and prey $x$ coexist in the form of a stable equilibrium (Fig. 6(b) and (c)). If this models a biological control process, then the success is dependent on the initial spread of control agent $z$. If the control agent is provided in small quantity, partial success can be achieved (in the sense that the pest $y$ is

(a)

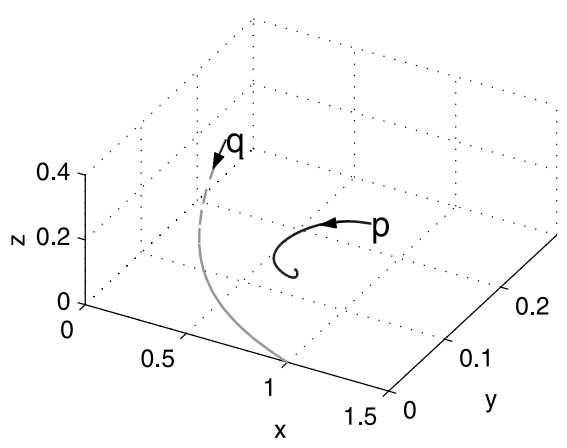

(c)



(b)

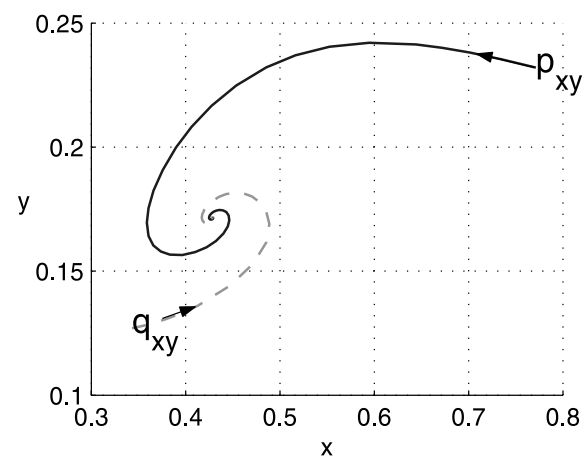

(d)

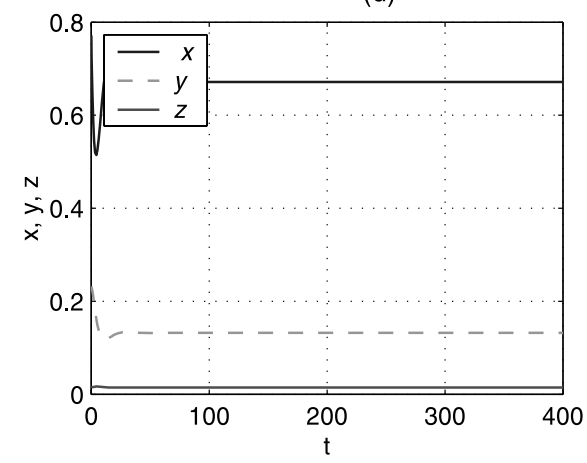

Fig. 6. In (a), the solution of system (2.1)-(2.3) starting at $p=(0.7714,0.232,0.0147)$ (near $\left.E_{c}\right)$ tends to $E_{c}$ (see also (d)), while the one starting at $q=(0.3433,0.1270,0.3670)$ tends to the steady state $(1,0,0)$ (see also (c)). Here, $m_{1}=1.4$, $m_{2}=10 / 9, d_{1}=d_{2}=1, c_{2}=1.7$ and $c_{1}=2 . E_{c}=\left(x_{c}, y_{c}, z_{c}\right)=(0.6714,0.1320,0.0147)$ is shown to be locally asymptotically stable (d). We see that (4.14) holds, hence $E_{100}$ is asymptotically stable. (b) Shows that in this case, when the top predator is absent, predator $y$ and prey $x$ coexist in the form of a stable equilibrium. Here $p_{x y}=(0.7714,0.2320,0)$ and $q_{x y}=(0.3433,0.1270,0)$. 
(a)

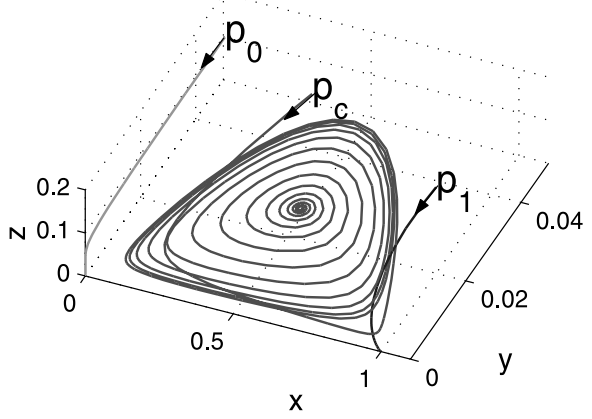

(c)

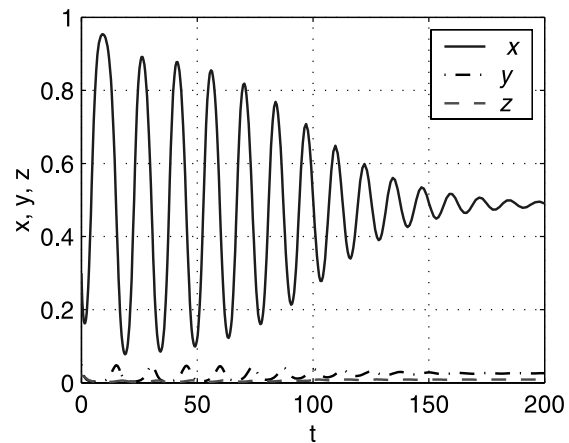

(b)

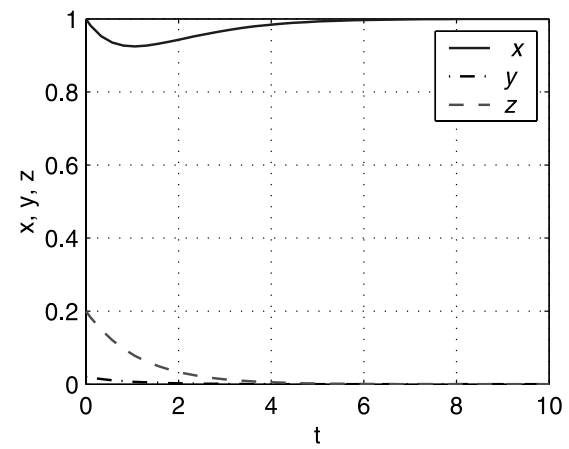

(d)



Fig. 7. When $d_{1}=7, d_{2}=1, m_{2}=4 / 3, m_{1}=7.895, c_{1}=133 / 13, c_{2}=2$, we have $E_{c}=(0.4881,0.0257,0.0086)$ and the conditions of Theorem 4.2 are satisfied. Hence (see (a)) there are solutions (starting at $p_{c}=(0.3,0.05,0.02)$, see also (c)) tend to $E_{c}$, some (starting at $p_{0}=(0.005,0.05,0.1)$, see also (d)) tend to the origin and yet some others (starting at $p_{1}=(1,0.02,0.2)$, see also $\left.(\mathrm{b})\right)$ tend to $(1,0,0)$.

suppressed but not eliminated, while $x$ is enhanced). Large quantity of $z$ ensures the eradication of pest $y$. This is a scenario where the pest $y$ is not so voracious, and the suitable control agent $z$ can suppress it, or drive it to extinction.

Notice that both Figs. 5 and 6 illustrate scenarios of bistability. One naturally wonders whether tri-stability scenarios are possible for model (2.1)-(2.3). A careful examination of the conditions that ensure the attractivity of the origin, $(1,0,0)$ and $E_{c}$ suggests that they maybe compatible for some set of parameters. This possibility is rigorously proven in the next theorem and is illustrated in Fig. 7.

Theorem 4.2. Assume that $m_{2}>d_{2}, c_{1}>1+d_{1}+c_{2}$ and

$$
\frac{\left(d_{1}+c_{2}\left(m_{2}-d_{2}\right) / m_{2}\right)^{2}}{d_{1}+c_{2}\left(\left(m_{2}-d_{2}\right) / m_{2}\right)^{2}}<m_{1}<\min \left\{1+d_{1}, c_{2}-d_{2}+d_{1},\left(d_{1}+c_{2} \frac{m_{2}-d_{2}}{m_{2}}\right) \sqrt{\frac{c_{1}}{c_{1}-1}}\right\},
$$

then system (2.1)-(2.3) has at least three attractors: $(0,0,0),(1,0,0)$ and $E_{c}$.

Proof. Since $\left(c_{1} / c_{1}-1\right)>1$, we have $\left(c_{1} / c_{1}-1\right)>\left(c_{1} / c_{1}-1\right)^{1 / 2}$. Hence the assumptions of Propositions 4.1 and 4.2 are satisfied. Therefore, both $(0,0,0)$ and $(1,0,0)$ are attractors. It remains to show that $E_{c}$ exists and is locally asymptotically stable. Since 


$$
m_{1}>\left(d_{1}+c_{2} \frac{m_{2}-d_{2}}{m_{2}}\right)^{2} /\left(d_{1}+c_{2}\left(\frac{m_{2}-d_{2}}{m_{2}}\right)^{2}\right)>d_{1}+c_{2} \frac{m_{2}-d_{2}}{m_{2}},
$$

we have $A=m_{1} /\left(d_{1}+c_{2} \frac{m_{2}-d_{2}}{m_{2}}\right)>1$. Since

$$
m_{1}<\left(d_{1}+c_{2} \frac{m_{2}-d_{2}}{m_{2}}\right) \sqrt{c_{1} /\left(c_{1}-1\right)}<\left(d_{1}+c_{2} \frac{m_{2}-d_{2}}{m_{2}}\right) \frac{c_{1}}{c_{1}-1},
$$

we see that $A\left(c_{1}-1\right)<c_{1}$ which implies that $0<c_{1}<A /(A-1)$. From Lemma 2.1, we conclude that the equilibrium $E_{c}$ exists. Moreover, from the proof of Proposition 3.1 and the facts

$$
\begin{aligned}
& m_{11}<0 \Longleftrightarrow d_{1}+c_{2} \frac{m_{2}-d_{2}}{m_{2}}<m_{1}<\left(d_{1}+c_{2} \frac{m_{2}-d_{2}}{m_{2}}\right) \sqrt{c_{1} /\left(c_{1}-1\right)}, \\
& m_{22}<0 \Longleftrightarrow\left(d_{1}+c_{2} \frac{m_{2}-d_{2}}{m_{2}}\right)^{2} /\left(d_{1}+c_{2}\left(\frac{m_{2}-d_{2}}{m_{2}}\right)^{2}\right)<m_{1},
\end{aligned}
$$

we see that $E_{c}$ is locally asymptotically stable and the theorem follows.

\section{Discussion}

Although numerous non-linear population models have been proposed and studied in the literature, most of them are build on the classical Lotka-Volterra platform which is incapable of describing both the vast biodiversity that we are part of and the massive extinction that we are now confronting. This is likely due to the fact that conventional ecological studies are often conducted in a small and controllable scale and thus enables the researchers to make the implicit assumption that populations are quasi-stable or near a stable steady state. Hence, most of the existing theoretical works on such mathematical models are centered around stability issues of possible positive steady states (coexisting equilibria). In other words, extinction dynamics is often overlooked or avoided. As a result, most models of population interactions are incapable of producing extinctions at lower levels that typically trigger the massive extinctions that we are witnessing today. In the context of biological control, rich extinction dynamics of any plausible model is especially desirable.

A valid ratio-dependent model formulation usually requires that the habitat of the interacting species is relatively small and free of refugees [13]. The continual fragmentation and shrinkage of habitats is often viewed as the main causes of massive extinctions of populations. Also, biological control is often successfully implemented in a relatively small area. This makes ratio-dependent based population models relevant and appealing due to their rich extinction dynamics. Our work on the ratio-dependent simple food chain (2.1)-(2.3) suggests that ratio-dependence is a plausible mechanism that contributes to both the massive extinctions of populations and the successful implementations of biological controls.

Extensive simulation of model (2.1)-(2.3) confirms the notion that the introduction of a natural enemy $z$ for pest $y$ helps the population level of prey $x$. This is illustrated by Fig. 4 . Without top predator, prey is severely depressed (Fig. 4(d)). With a medium aggressive (medium $c_{2}$ values) top 
predator, species may coexist in a stable form with enhanced prey and depressed pest population levels (see Fig. 4(c)). A more voracious top predator may slightly increase further the average prey level and decrease average pest level at the expense of destabilizing such stable coexistence (see Fig. 4(b)).

Our simulation work also shed light to the following interesting question: assume that a voracious pest $y$ (defined as any pest (predator) species that capable of driving its host (prey) to extinction) invades a host $x$, can one introduce a suitable top predator to ensure a meaningful (with some success) biological control? Fig. 5 suggests the answer can be positive. In such situations, it maybe necessary to introduce the control agent swiftly and forcefully (see the solution starts at $p$ ) in order to slow down and or even stop the extinction of $x$. A delayed action can mean total failure (see the solution starts at $q$ ).

Our extinction results suggest that biological control is possible under various scenarios. However, in practice, we often want to reduce the pest $y$ to an acceptable level in a finite time. In order to accomplish that, we need to give a estimate as how much $z$ shall be introduced to control $y$. Clearly, theoretical findings are powerless for this particular practical concern. Nevertheless, such an estimation work can be meaningfully conducted by some carefully designed simulations of model (2.1)-(2.3).

A most startling finding of this paper is the discovery of a tri-stability scenario described in Theorem 4.2. To the best of our knowledge, such sensitivity, although may very well exist in other low-dimensional (two- or three-dimensional autonomous ODE models) models, has never been identified explicitly in the literature. Extensive simulation on the model (2.1)-(2.3) with the set of parameters that generates Fig. 7 shows that if we fix the initial population levels for $x$ and $y$, small values of $z(0)$ often ensure the solutions tend to the coexistence steady state $E_{c}$. Increasing $z(0)$ significantly may lead to the pest free (or predator free) boundary steady state $(1,0,0)$, signaling the ultimate success of biological control. In other word, it pays to introduce more control agents $z$. If the crop $x$ is severely infested (high values of $y(0) / x(0)$ ), then the crop is doomed regardless of the amount of control agent one may introduce. This confirms the need of early planning of a biological control.

What remains particularly intriguing is whether model (2.1)-(2.3) is capable of generating chaotic dynamics similar to that produced by Lotka-Volterra based simple food chain model [27]. Our extensive simulation on the model with $m_{1}=10, m_{2}=2, d_{1}=1, d_{2}=1, c_{1}=1, c_{2}=11$ indicating an affirmative answer is highly likely (see Fig. 8(b)). More works on this (such as a thorough bifurcation study and the calculation of the Lyapunov exponent of this chaotic looking solution) are needed.

Another mathematically interesting question remains open for model (2.1)-(2.3) is under what conditions, all three species coexist regardless of initial conditions. This is the so-called persistence question. Global stability of the positive equilibrium will ensure this but is even more difficulty to work on. Persistence results for two trophic level ratio-dependent predator-prey models with or without time delay are available $[16,35,36]$. On the other hand, persistence issue is less relevant for biological control applications, since the most desired outcome is a pest free steady state.

Model (2.1)-(2.3) is most suitable when biological control agent is a natural predator of the pest. Other models may also be plausible for this situation. For example, one may use the classical prey-dependent functional response $x y /\left(a_{1}+x\right)$ for pest and its numerical response, while using ratio-dependent functional response $y z /\left(y+a_{2} z\right)$ for top predator and its numerical response. This 
(a)


(b)
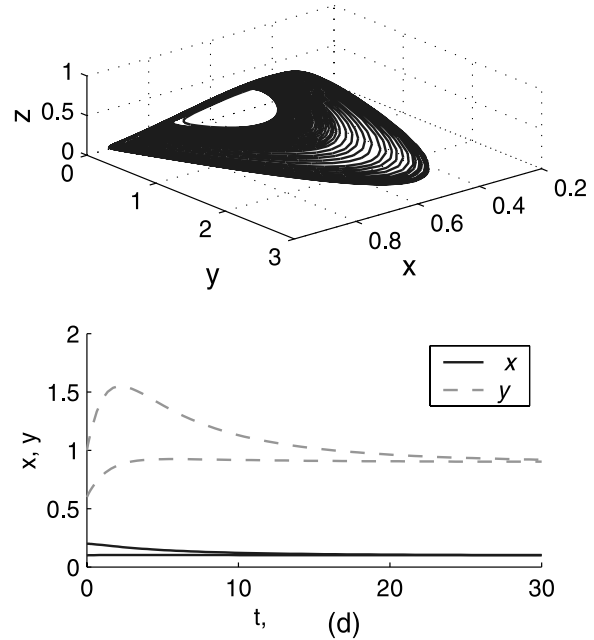

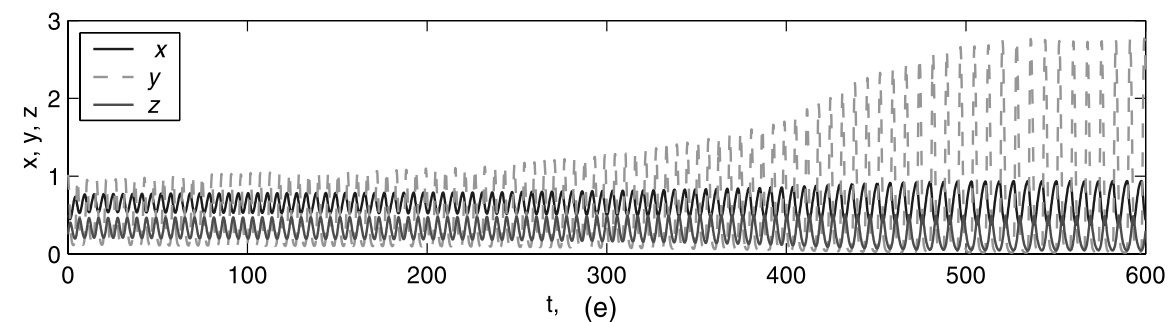

Fig. 8. In this figure, as in Fig. $4, m_{1}=10, m_{2}=2, d_{1}=1, d_{2}=1, c_{1}=1, c_{2}=11$. (a) Depicts the $x y$-plane projections of two solutions (starting at $p$ and $q$ ) of system (2.1)-(2.3), one (starting at $p$ ) of which is the periodic solution depicted in Fig. 4(a). For this set of parameters, $E_{c}$ exists and is unstable. (b) Highlights one of the solution which appears to be chaotic in a three-dimensional view. (c) Depicts time course of this chaotic looking solution.

is particular suitable when pest is uniformly distributed and slow moving. This mixed selection of functional response yields a model of the following form:

$$
\begin{aligned}
x^{\prime}(t) & =r x\left(1-\frac{x}{K}\right)-\frac{1}{\eta_{1}} \frac{m_{1} x y}{a_{1}+x}, \quad x(0)>0, \\
y^{\prime}(t) & =\frac{m_{1} x y}{a_{1}+x}-d_{1} y-\frac{1}{\eta_{2}} \frac{m_{2} y z}{a_{2} z+y}, \quad y(0)>0, \\
z^{\prime}(t) & =\frac{m_{2} y z}{a_{2} z+y}-d_{2} z, \quad z(0)>0,
\end{aligned}
$$

where the meanings of the parameters are self-evident. This model may also be capable of admitting a pest free attractor. If parasite is chosen as the control agent, then quite different model maybe called for. A plausible model may build a simple infection mechanism on top of a typical predator-prey model with either prey-dependent or ratio-dependent (or the more general predator-dependent ones) functional responses. The model can take various forms depending on the specific choices of infection mechanisms and the predator functional responses. Appropriately 
formulated, such models shall also be able to generate rich extinction dynamics. An example of such models with prey-dependent functional response may take the form

$$
\begin{aligned}
& x^{\prime}(t)=r x\left(1-\frac{x}{K}\right)-\frac{c_{1} x(y+z)}{a+x}, \quad x(0)>0, \\
& y^{\prime}(t)=\frac{m_{1} x(y+f z)}{a+x}-d y-\frac{c_{2} y z}{y+z}, \quad y(0)>0, \\
& z^{\prime}(t)=\frac{m_{2} y z}{y+z}-(d+\alpha) z, \quad z(0)>0 .
\end{aligned}
$$

The infection mechanism is the same one adopted by Ebert et al. [34] (but was erroneously implemented in their model, causing the failure of generating deterministic extinction scenarios that needed to explain such phenomena in the fields (see [37]). This mechanism can be used to model a microparasite transmission for a horizontally transmitted parasite that reduces fecundity and survival of its host. In this case, the plausible meanings of the parameters are again clear. It will be interesting to know if the naturally occurring ratio-dependence (resulted from the infection mechanism) in the uninfected $(y)$ and the infected pest $(z)$ equations will generate rich extinction dynamics that naturally account for the various deterministic extinction scenarios observed in the fields.

\section{Acknowledgement}

This work is partially supported by NSF grant DMS-0077790 and is initiated, while visiting the National Center for Theoretical Science, Tsing Hua University, Hsinchu, Taiwan ROC.

\section{References}

[1] P.A. Abrams, The fallacies of 'ratio-dependent' predation, Ecology 75 (1994) 1842.

[2] P.A. Abrams, L.R. Ginzburg, The nature of predation: prey dependent, ratio-dependent or neither? Trends Ecol. Evol. 15 (2000) 337.

[3] H.I. Freedman, Deterministic Mathematical Models in Population Ecology, Marcel Dekker, New York, 1980.

[4] R.M. May, Stability and Complexity in Model Ecosystems (Princeton Landmarks in Biology), Princeton University, Princeton, NJ, 2001.

[5] N.G. Hairston, F.E. Smith, L.B. Slobodkin, Community structure, population control and competition, Am. Nat. 94 (1960) 421.

[6] M.L. Rosenzweig, Paradox of enrichment: destabilization of exploitation systems in ecological time, Science 171 (1969) 385.

[7] P.A. Abrams, C.J. Walters, Invulnerable prey and the paradox of enrichment, Ecology 77 (1996) 1125.

[8] R.F. Luck, Evaluation of natural enemies for biological control: a behavior approach, Trends Ecol. Evol. 5 (1990) 196.

[9] R. Arditi, A.A. Berryman, The biological control paradox, Trends Ecol. Evol. 6 (1991) 32.

[10] G.F. Gause, The Struggle for Existence, Williams \& Wilkins, Baltimore, MD, 1934.

[11] R. Arditi, L.R. Ginzburg, H.R. Akcakaya, Variation in plankton densities among lakes: a case for ratio-dependent models, Am. Natural 138 (1991) 1287.

[12] H.R. Akcakaya, R. Arditi, L.R. Ginzburg, Ratio-dependent prediction: an abstraction that works, Ecology 76 (1995) 995. 
[13] C. Cosner, D.L. DeAngelis, J.S. Ault, D.B. Olson, Effects of spatial grouping on the functional response of predators, Theor. Pop. Biol. 56 (1999) 65.

[14] R. Arditi, L.R. Ginzburg, Coupling in predator-prey dynamics: ratio-dependence, J. Theor. Biol. 139 (1989) 311.

[15] S.B. Hsu, T.W. Hwang, Y. Kuang, Global analysis of the Michaelis-Menten type ratio-dependence predator-prey system, J. Math. Biol. 42 (2001) 489.

[16] E. Beretta, Y. Kuang, Global analyses in some delayed ratio-dependent predator-prey systems, Nonlinear Anal., TMA 32 (1998) 381.

[17] C. Jost, O. Arino, R. Arditi, About deterministic extinction in ratio-dependent predator-prey models, Bull. Math. Biol. 61 (1999) 19.

[18] F. Berezovskaya, G. Karev, R. Arditi, Parametric analysis of the ratio-dependent predator-prey model, J. Math. Biol. 43 (2001) 221.

[19] D. Xiao, S. Ruan, Global dynamics of a ratio-dependent predator-prey system, J. Math. Biol. 43 (2001) 268.

[20] H.I. Freedman, R.M. Mathsen, Persistence in predator-prey systems with ratio-dependent predator-influence, Bull. Math. Biol. 55 (1993) 817.

[21] S.-B. Hsu, T.-W. Hwang, Global stability for a class of predator-prey systems, SIAM J. Appl. Math. 55 (1995) 763.

[22] S.B. Hsu, T.W. Hwang, Hopf bifurcation for a predator-prey system of Holling and Leslie type, Taiwanese J. Math. 3 (1999) 35.

[23] S.B. Hsu, T.W. Hwang, Y. Kuang, Rich dynamics of a ratio-dependent one prey two predator model, J. Math. Biol. 43 (2001) 377.

[24] H.I. Freedman, P. Waltman, Mathematical analysis of some three-species food-chain models, Math. Biosci. 33 (1977) 257.

[25] C.H. Chiu, S.B. Hsu, Extinction of top predator in a three level food-chain model, J. Math. Biol. 37 (1998) 372.

[26] A. Hastings, T. Powell, Chaos in a three-species food chain, Ecology 72 (1991) 896.

[27] A. Klebanoff, A. Hastings, Chaos in three species food chains, J. Math. Biol. 32 (1993) 427.

[28] A. Klebanoff, A. Hastings, Chaos in one-predator, two-prey models: general results from bifurcation theory, Math. Biosci. 122 (1994) 221.

[29] K. McCann, P. Yodzis, Bifurcation structure of a three-species food chain model, Theor. Pop. Biol. 48 (1995) 93.

[30] Yu.A. Kuznetsov, S. Rinaldi, Remarks on food chain dynamics, Math. Biosci. 134 (1996) 1.

[31] S. Muratori, S. Rinaldi, Low- and high-frequency oscillations in three-dimensional food chain systems, SIAM J. Appl. Math. 52 (1992) 1688.

[32] H.I. Freedman, J.W.-H. So, Global stability and persistence of simple food chains, Math. Biosci. 76 (1985) 69.

[33] Y. Kuang, Global stability and persistence in diffusive food chains, ANZIAM J. 43 (2001) 247.

[34] D. Ebert, M. Lipstich, K.L. Mangin, The effect of parasites on host population density and extinction: experimental epidemiology with Daphnia and six microparasites, Am. Natural 156 (2000) 459.

[35] Y. Kuang, E. Beretta, Global qualitative analysis of a ratio-dependent predator-prey system, J. Math. Biol. 36 (1998) 389.

[36] Y. Kuang, Rich dynamics of Gause-type ratio-dependent predator-prey system, Fields Inst. Commun. 21 (1999) 325.

[37] T.W. Hwang, Y. Kuang, Deterministic extinction effect of parasites on host populations, J. Math. Biol., in press. 